\title{
DESARROLLO DE UN DISPOSITIVO DE ADQUISICIÓN DE DATOS PARA EL ESTUDIO Y CARACTERIZACIÓN DE CALEFONES SOLARES CONSTRUIDOS CON ALUMNOS DE LA FACENA PARA SU INSTALACIÓN EN ZONAS RURALES.
}

Rolando L. BENÍTEZ ALONSO; Dra. Noemí SOGARI; Lic. Eduardo J. RICCIARDI(*)

Palabras claves: calefón solar, electrónica, adquisición de datos.

RESUMEN: el presente trabajo expone los avances alcanzados respecto al diseño y desarrollo de un dispositivo de adquisición de datos para la caracterización termodinámica de calefones solares. El objetivo del sistema es monitorear los parámetros relevantes para analizar el rendimiento térmico de un calefón solar y almacenarlos en una base de datos. El diseño del sistema se basa en la implementación de un microcontrolador en plataforma de arduino, sensores debidamente calibrados para la obtención de mediciones de gran precisión, un protocolo de comunicación inalámbrica entre el dispositivo y una central de monitoreo y una interfaz gráfica de usuario encargada del tratamiento de datos medidos.

Actualmente, el trabajo se encuentra en etapa de desarrollo, se realizaron las primeras programaciones del microcontrolador y prueba de módulos electrónicos y sensores.

\section{INTRODUCCIÓN.}

Las fuentes de energías renovables se han convertido en alternativas, de bajo costo, para el desarrollo de aplicaciones que favorecen el cuidado del medio ambiente y la economía local. La construcción de calefones solares con materiales de bajo costo ha permitido poner al alcance de diversas familias, que no pueden contar con suministros de gas o energía eléctrica, el aprovechamiento de la energía solar para el calentamiento de agua.

El crecimiento de la utilización de estos dispositivos ha hecho imperativa la innovación tecnológica de los mismos para mejorar su rendimiento. El estudio de sus características permite mejorar aspectos constructivos para obtener una mayor eficiencia de funcionamiento y construir equipos de mejor calidad.

La electrónica aparece como el complemento ideal para la caracterización de dispositivos. La amplia gama de sensores y mi-

$\left(^{*}\right)$ Grupo de Investigación, Desarrollo y Transferencia de Energías Sustentables y del Medio Ambiente (GIESMA).

Facultad de Ciencias Exactas y Naturales y Agrimensura (FACENA) Universidad Nacional del Nordeste (UNNE) Av. Libertad 5460 C.P. 3400 - Corrientes. Tel. +54 0379-4473931 int. 134 - Fax +54 0379-4473930.

Celular: 0379-4765232. Correo: rololeone188@gmail.com. 


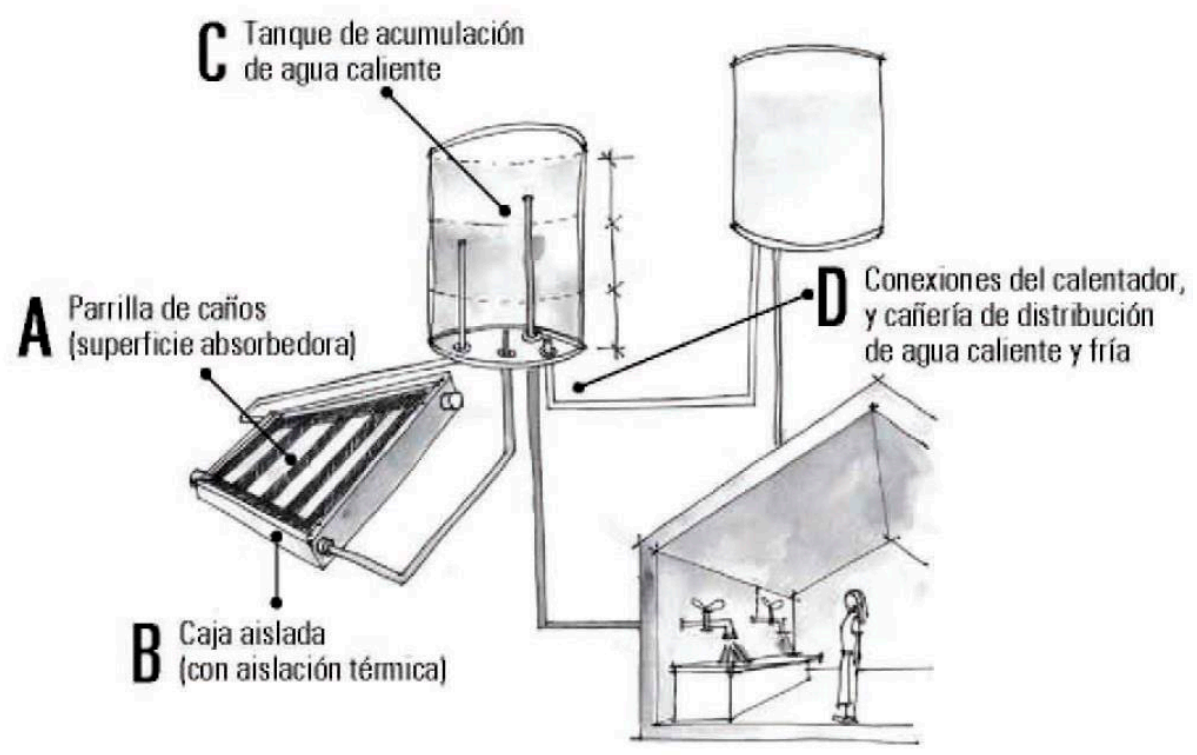

Figura 1: Esquema representativo del sistema. (Facultad de Arquitectura y Urbanismo. Universidad Nacional de la Plata)

crocontroladores existentes en el mercado ofrece interesantes alternativas respecto a calidad y precios para el diseño y construcción de dispositivos de adquisición de datos para el estudio de los calefones solares, siendo este el objetivo principal del trabajo.

Existen numerosos aparatos electrónicos en el mercado, que permiten realizar distintas mediciones de diferentes magnitudes físicas, aunque generalmente resultan ser un poco costosos y tal vez no posean todas las funciones requeridas para el estudio. De ahí que la construcción de un dispositivo propio de adquisición de datos, con todos los requerimientos necesarios y a un costo accesible, se convierte en una gran alternativa para la ejecución del trabajo.

\section{CALEFÓN SOLAR.}

El funcionamiento del dispositivo se basa en el aprovechamiento de la energía calórica proveniente de la radiación infrarroja. Los colectores funcionan principalmente en base al espectro visible e infrarrojo del espectro solar.

E1 colector solar se compone básicamente de una caja o marco con aislamiento, una cubierta de vidrio o de plástico que será la ventana por donde ingresa la radiación solar, una placa absorbedora y una red de cañerías de color oscuro (Figura 1). La radiación solar es absorbida por la placa que está construida de un material que transfiere rápidamente el calor a un fluido que circula a través de tubos. El agua fría ingresa a la red de cañerías del colector desde el tanque, por acción de la gravedad, se calienta mediante los absorvedores y reingresa al tanque con una temperatura más alta. Este proceso se lleva a cabo de manera continua.

La cubierta transparente transmite una gran cantidad de la luz de onda corta del 
espectro solar y al mismo tiempo, sólo deja pasar muy poca radiación de onda larga, que es el calor emitido por la superficie absorbedora, produciendo un efecto invernadero. Además, la cubierta evita que el viento pueda disipar, por convección, el calor colectado. Junto al marco ofrecen protección de las condiciones ambientales adversas.

La aislación del sistema de captación de calor reduce las pérdidas de calor. La caja, debidamente aislada, debe estar orientada en forma perpendicular a los rayos del sol.

El agua calentada por el colector se acumula en un tanque independiente deberá estar debidamente aislado para evitar que el agua se enfríe durante la noche.

Una ventaja importante de estos equipos es que no necesita un equipo de mantenimiento ni cuidado especial. Otra ventaja es la de poder utilizar directamente agua corriente de la red de suministro de agua potable.

Debido a las características constructivas del colector no se debe jamás dejar el mismo sin agua en su interior, ya que pondría en riesgo los materiales utilizados, dificultando su buen funcionamiento futuro.

\section{DISPOSITIVO DE ADQUISI- CIÓN DE DATOS.}

Una de las funciones del dispositivo de adquisición de datos es el monitoreo constante y en tiempo real del funcionamiento del calefón solar. El equipo monitorea la temperatura ambiente, presión atmosférica, humedad relativa, temperatura de la superficie del colector solar, la temperatura de entrada y salida del agua, el caudal de agua y la radiación incidente sobre la superficie colectora.
El diseño y la construcción del dispositivo de adquisición de datos se llevaron a cabo en torno a una plataforma Arduino, específicamente el Arduino MEGA 2560. Es uno de los modelos más potentes del mercado, ofreciendo gran capacidad de procesamiento y un buen número de periféricos de entrada y salida a un buen precio. Cabe mencionar que es un dispositivo con una programación sencilla y de fácil adquisición en el mercado.

Para el registro de las variables supra mencionadas, se utilizan distintos tipos de sensores.

Los datos registrados se almacenan en una tarjeta de memoria Micro-SD. Esto permite tener una base de datos de todas las mediciones que se lleven a cabo en diferentes fechas y en distintas horas del día. También le otorga gran flexibilidad al sistema, ya que se puede retirar la tarjeta de memoria para llevarla a una computadora y trabajar con los datos obtenidos o intercambiarla por otra. De esta manera, no es necesario desmontar todo el dispositivo de medición del colector solar.

El sistema cuenta con un módulo de wifi que se encarga de transmitir los datos obtenidos, mediante una conexión de internet a una estación central de monitoreo.

Se prevé además el desarrollo, mediante el software Matlab, de una interfaz gráfica de usuario con el cual se llevarán a cabo los distintos cálculos y la obtención de curvas representativas, que se consideren convenientes.

Sensor digital de temperatura DS18B20: permite medir temperaturas 
desde $-55^{\circ} \mathrm{C}$ hasta $125^{\circ} \mathrm{C}$, su encapsulado sellado permite protegerlo de la intemperie y sumergirlo en agua, es de tamaño pequeño, del orden de los $2 \mathrm{~cm}$ y tiene una precisión de $\pm 0,5^{\circ} \mathrm{C}$. Son sensores de gran calidad. Con este dispositivo se desarrolla una matriz de sensores, que se dispondrá sobre el colector solar a efectos de medir la temperatura de la superficie de la cubierta transparente y tener registros de los valores que pueden llegar a alcanzar durante todo un día de funcionamiento. También se mide la temperatura del agua a la entrada y salida de la red de tuberías del colector.

Sensor de temperatura LM35: precisión calibrada de $1^{\circ} \mathrm{C}$ cuyo rango medición está comprendido entre $-55^{\circ} \mathrm{C}$ y $150^{\circ} \mathrm{C}$, ofrece una respuesta lineal de $10 \mathrm{mV} /{ }^{\circ} \mathrm{C}$ (milivoltios/grados Celsius), la tensión de salida del dispositivo es proporcional a la temperatura. Se utiliza para la medición de temperatura ambiente. Su linealidad de operación permite realizar mediciones con una gran exactitud y con gran facilidad.

Sensor de humedad relativa $\mathrm{HiH}-4000-$ 001: de tamaño reducido, del orden de los $2,5 \mathrm{~mm}$, de respuesta rápida, presenta un rango de temperatura de operación entre $-40^{\circ} \mathrm{C}$ y $85^{\circ} \mathrm{C}$, rango de humedad relativa de funcionamiento de $0 \%$ a $100 \%$ y una precisión de humedad relativa de $\pm 3.5 \%$. Se utiliza para la medición de humedad relativa.

Sensor de presión atmosférica SCX30DN: son de gran precisión, permiten realizar mediciones de presión diferencial y absoluta de hasta 30psi. Puede operar en un rango de temperaturas de $0^{\circ} \mathrm{C}$ hasta $70^{\circ} \mathrm{C}$ por lo que debe estar resguardado para evitar sobrecalentamientos y mediciones erróneas. Se utiliza para la medición de presión atmosférica.

Los sensores LM35, HiH-4000-001 y SCX30DN permiten establecer las condiciones climáticas bajo las cuáles se llevan a cabo las mediciones para la evaluación del comportamiento del sistema.

Módulo de tarjeta micro SD: nos permite insertar una memoria micro SD que son las más comunes en el mercado, el módulo se puede alimentar con $3.3 \mathrm{~V}$ o $5 \mathrm{~V}$ usando los pines respectivos. Arduino cuenta con una librería para usar estas memorias, que funciona con varios módulos. La librería ya viene integrada al entorno de desarrollo interactivo (IDE), por lo que no necesitamos instalar ni descargar nada.

Módulo ZS-042, RTC3231: incluye un integrado DS3231, un RTC de alta precisión que emplea para comunicarse un bus serie del tipo $\mathrm{I}^{2} \mathrm{C}$ ("I cuadrado C"). Este chip no solo lleva la cuenta de la hora, sino también de la fecha (día, mes, año y día de la semana) haciendo los ajustes necesarios según la cantidad de días de cada mes e incluso los años bisiestos. Es capaz de seguir funcionando con una tensión de 3 Voltios consumiendo muy poca corriente, lo que le permite usar una batería como alimentación, de manera de poder seguir llevando la hora aun cuando se apaga el equipo en el que está conectado. Este módulo permite realizar mediciones de tiempo para registrar el momento del día en el que se realizan las mediciones.

Módulo wifi esp8266: para enviar los datos registrados a una central de monito- 

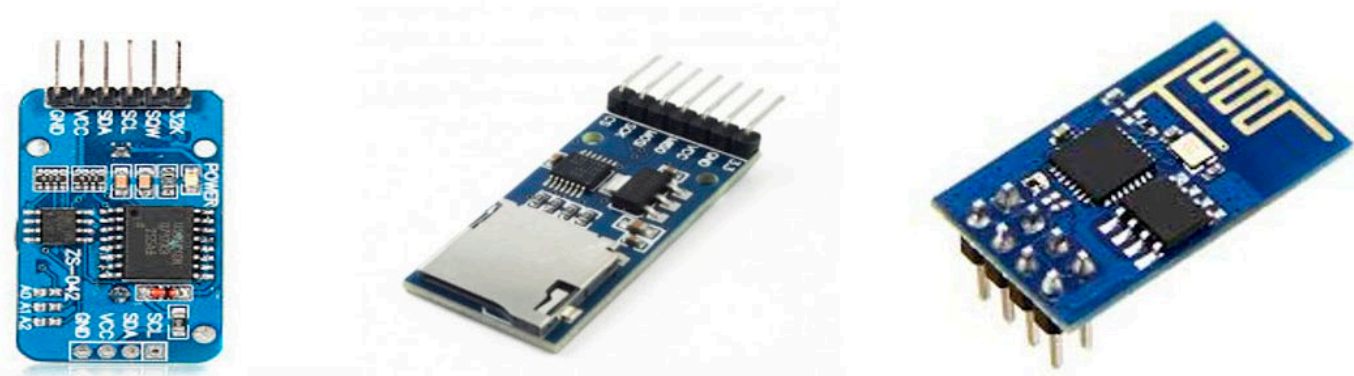

Figura 2: módulos electrónicos para Arduino.

a-Módulo wifi esp8266. b-Módulo RTC 3132. c-Módulo de tarjeta micro SD.

reo. Es un módulo muy sencillo que incluye todo lo necesario para conectarse a un punto de acceso WIFI mediante comandos de texto AT, vía una puerta serie, que puede ser configurada a diferentes velocidades. Una vez que se le instruye para que se conecte a nuestra WIFI, el módulo es capaz de enviar información que se le remite vía la puerta serie a una dirección IP y puerto que se desee.

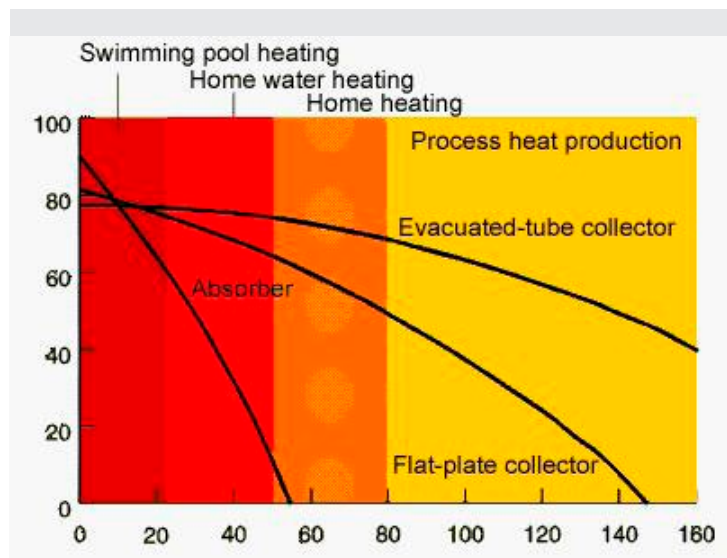

Figura 3: Variación de la eficiencia, en distintos tipos de colectores, en función de la diferencia de temperatura del agua entre la salida y la entrada del colector (Placco et al., 2008)

\section{EFICIENCIA DE UN COLECTOR SOLAR}

La eficiencia de un colector solar se define como el cociente de la energía térmica útil frente a la energía solar total recibida. Además de las pérdidas térmicas existen las pérdidas ópticas. El factor de conversión o eficiencia óptica indica que porcentaje de los rayos solares que penetran en la cubierta transparente del colector (la transmisión) es absorbido. Básicamente, es el producto de la tasa de transmisión de la cubierta y la tasa de absorción del absorbedor. (Placco et al., 2008)

\section{RENDIMIENTO}

El rendimiento del calefón solar, en función de las temperaturas de entrada y salida de agua será calculado en función de las mediciones que se realicen a lo largo del día con el dispositivo de adquisición de datos conectado al equipo.

MONITOREO DEL FUNCIONAMIENTO.

El dispositivo de adquisición de datos se conectará al calefón solar y gracias a su gran autonomía energética, podrá permanecer co- 


\section{Rendimiento $=\frac{\text { Flujo } * \text { Area transversal } *(\text { Temperatura Agua-Temperatura Ambiente })}{\text { Area Total de Colección } * \text { Irradiancia }}$ \\ Ecuación 1}

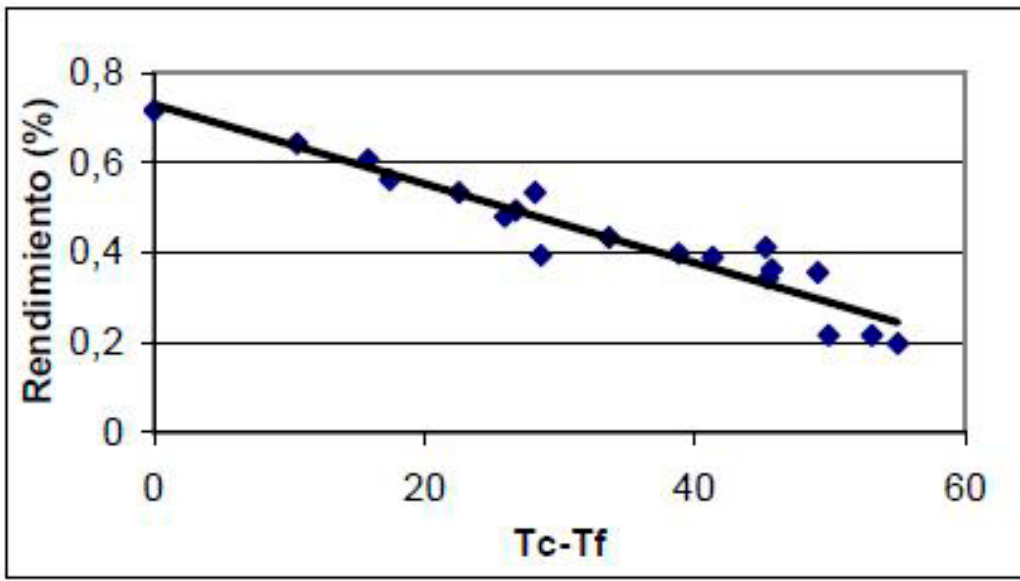

Figura 4: Rendimiento de un calefón solar completo de industria nacional. Colector (gentileza INNOVAR S.R.L.) Tc: Temperatura del agua a la salida del colector (caliente) Tf: Temperatura del agua al ingresar al colector (fría) (Placco et al., 2008)

nectado a lo largo de todo el día, enviando los datos directamente a la central de monitoreo y guardando todos los registros en una tarjeta de memoria micro SD, para su posterior procesamiento.

Se espera poder monitorear el funcionamiento completo del calefón solar, de modo a realizar un buen estudio y mejorar aspectos constructivos para la construcción de equipos de mejor calidad y un mejor aprovechamiento de energía.

\section{PRIMERAS PRUEBAS DEL DIS-} POSITIVO.

Las primeras pruebas se realizaron con sensores de temperatura y algunos módu- los de Arduino. Los ensayos consistieron en medir la temperatura ambiente, registrar la hora exacta en que se midió y guardar los registros en una memoria micro SD. Los datos pudieron visualizarse perfectamente en una computadora.

Una de las ventajas del módulo de tarjeta micro SD es que crea un archivo de extensión .txt para poder guardar en él todos los registros. Este tipo de archivos luego puede ser llevado a un computador para poder analizar los datos contenidos en ellos.

Se debe tener en cuenta que las pruebas de verificación de sensores y módulos se han realizado bajo condiciones favorables para los equipos electrónicos. Entiéndase 


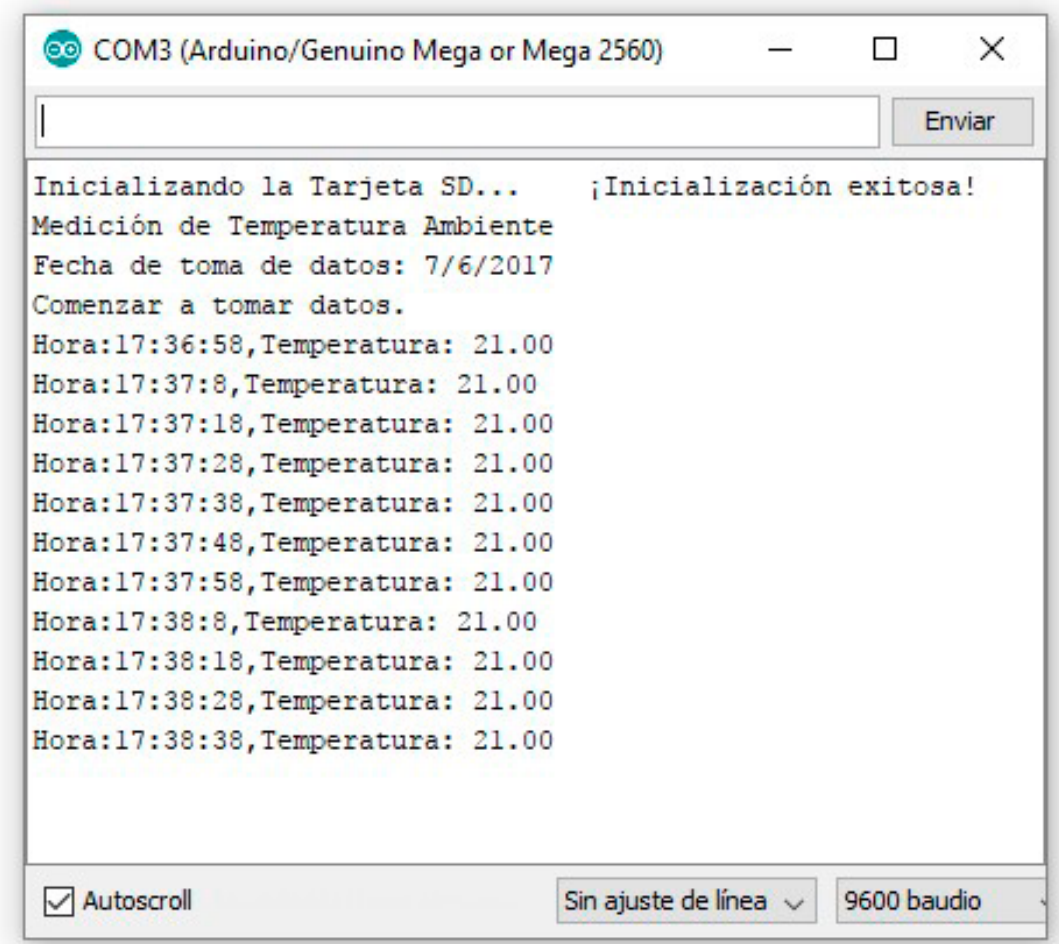

Figura 5: Ensayos de sensores y módulos a temperatura ambiente (21C aproximadamente).

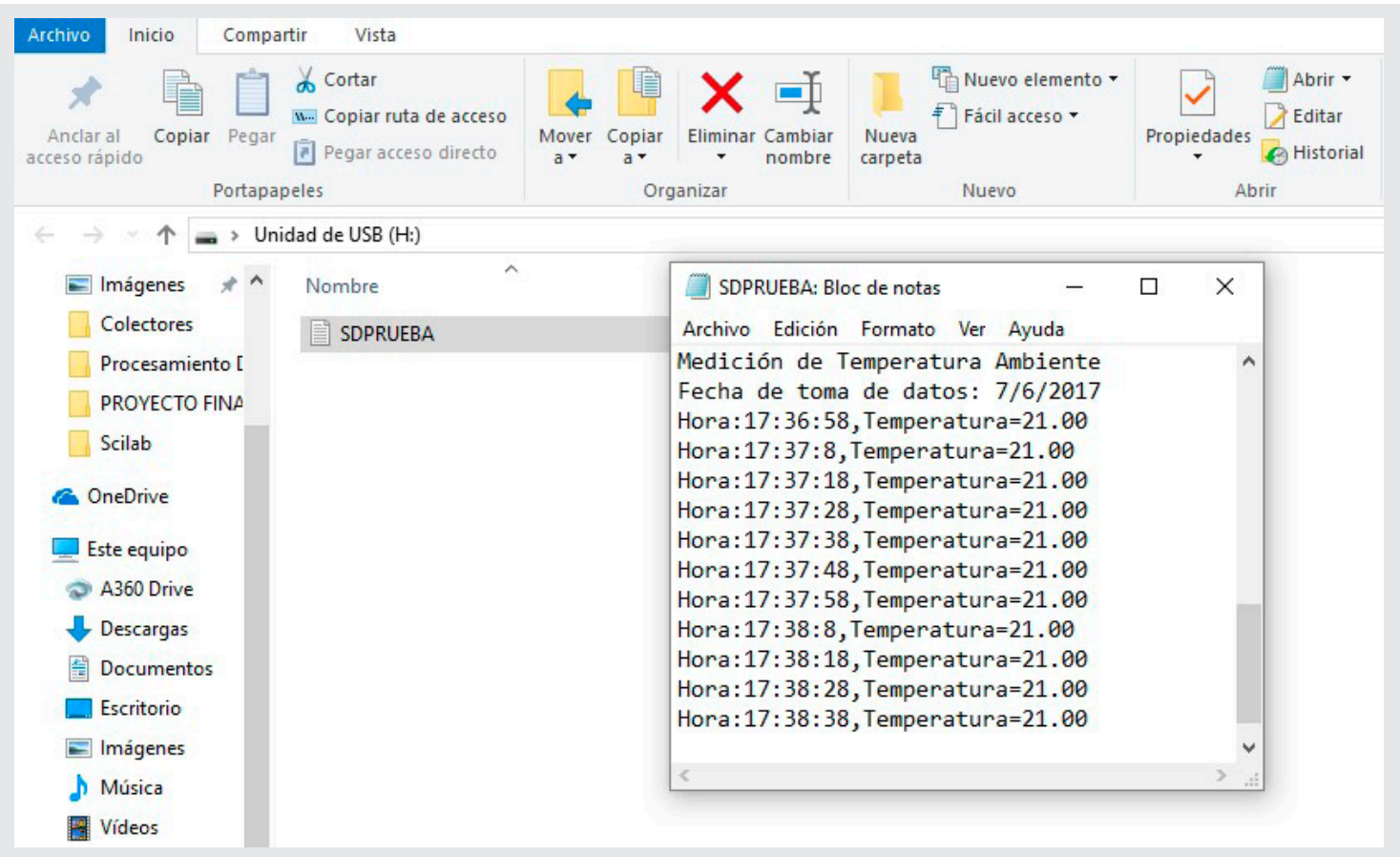

Figura 6: Ensayos de sensores y módulos registrados en el computador $\left(21^{\circ} \mathrm{C}\right.$ aproximadamente) 


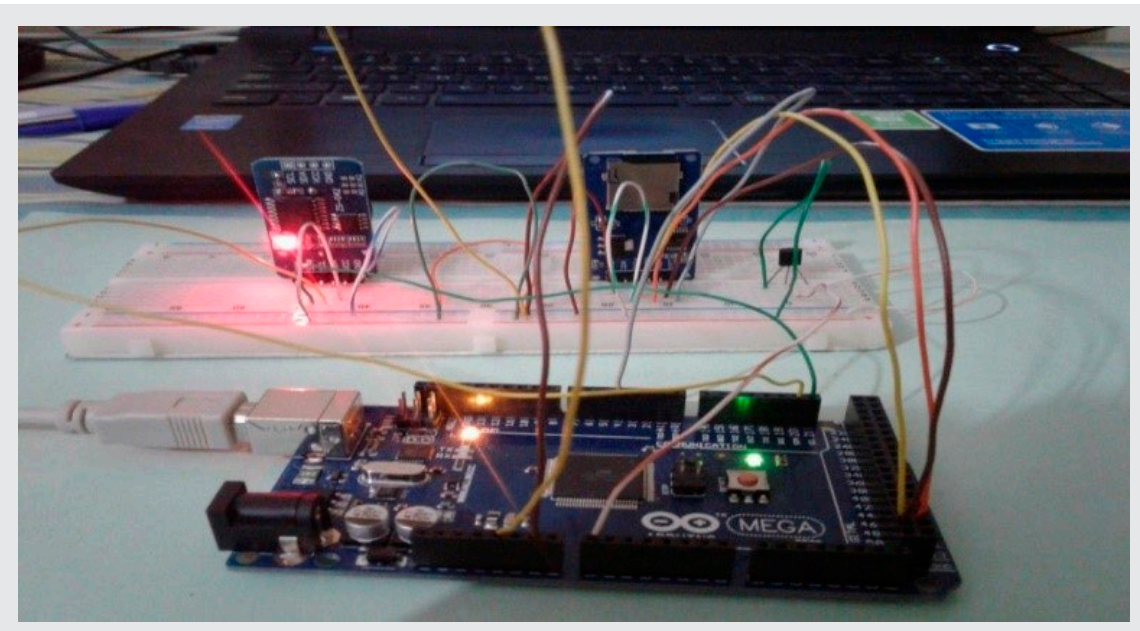

Figura 7: Pruebas de sensores y módulos a temperatura ambiente $\left(21^{\circ} \mathrm{C}\right.$ aproximadamente)

por condiciones favorables, como temperaturas muy por debajo de los límites de operación de los dispositivos, lugares bajo techo aislados de condiciones adversas que propicien un mal funcionamiento.

Una vez terminado el diseño y verificación del correcto funcionamiento de los componentes se debe llevar a cabo el montaje del prototipo y su posterior puesta en marcha.

\section{CONCLUSIÓN.}

El uso de dispositivos de adquisición de datos de bajo costo, tamaños reducidos y de una gran autonomía energética representa una gran ventaja en la caracterización de equipos en general. En este caso se lleva a cabo el diseño y construcción de un dispositivo que se ajusta a los parámetros que se desean medir. En el mercado no se encuentran dispositivos capaces de realizar todas estas mediciones en un mismo equipo, sino que se deben comprar varios equipos para medir diferentes parámetros, lo que implica un gran costo.

Arduino, nos presenta una gran gama de modelos y módulos capaces de facilitar las tareas y a un costo sumamente bajo. También cabe destacar la gran precisión de los sensores, pudiendo obtener mediciones de gran fidelidad.

Estimando gastos del equipo construido, el total rondaría los $A R \$ 2500$, recordar que se espera que el dispositivo pueda medir una gran variedad de magnitudes físicas, como irradiancia, humedad relativa, presión atmosférica, temperatura, además de contar con un sistema inalámbrico de transmisión de datos.

Podemos encontrar en el mercado equipos que solamente miden la radiación solar a precios muy costosos, tal vez no se adecuen a las características del equipo diseñado en cuestión. Otros dispositivos de adquisición de datos rondan los valores mínimos de AR $\$ 3000$ en adelante, pero no cuentan con todos los sensores que ne- 
cesitamos. Esto marca la importancia del dispositivo diseñado en cuanto a precios en el mercado y además, no menos importante, es que el diseño se realiza con el fin de ofrecer todas las facilidades posibles para un uso sencillo y confiable.

La importancia de estos equipos de adquisición de datos radica en la facilidad para medir características en el funcionamiento del equipo, transmitir la información directamente a un computador, realizar cálculos, levantar curvas y en base a resultados tomar decisiones y emitir conclusiones que ayuden a mejorar aspectos constructivos de los calefones solares.

Keywords: sensors, thermodynamic characterization, data acquisition.

\section{Abstract.}

This work shows the design and development of a data acquisition device for the thermodynamic characterization of solar water heaters. The objective is to monitor the relevant parameters for analyzing the thermal performance of a solar water heater and storing them in a database. The design of the system is based on the implementation of a microcontroller in arduino platform, sensors properly calibrated to obtain measurements of high precision, a protocol of wireless communication between the device and a central monitoring and a graphical user interface in charge of the treatment of measured data.

Currently, the work is in the development stage, with the first programming of the microcontroller and testing of electronic modules and sensors.

\section{REFERENCIAS Y BIBLIOGRAFÍA.}

Boylestad R.,NashelskyL.(2009).Electrónica:Teoría de Circuitos y Dispositivos Electrónicos. Décima edición. ISBN: 978-607-442-292-4. Calentador de Agua Solar. Manual del Usuario. Tecnología sencilla (2008). Facultad de Arquitectura y Urbanismo. Universidad Nacional de la Plata. Cora Placco, Luis Saravia, Carlos Cadena (2008). Colectores Solares para Agua Caliente. INENCO, UNSa, CONICET. Salta. De la Horra Kïllmer, M. (2013). Proyecto de Fin de Carrera: Sistema de Adquisición de Datos basado en la plataforma Arduino. Aplicaciones a Matlab, Simulink y Android. Universidad Carlos III de Madrid. Leganés - España. Duffie J., Beckman W. (1980). Solar Engineering of Thermal Processes. John Wiley \& Sons. New York.

Enríquez Herrador, R. (2009). Guía de Usuario de Arduino. Universidad de Córdoba. España. Fernández Díez P. (2003). Procesos Termosolares en baja, media y alta temperatura. Departamento d e Ingeniería Eléctrica y Energética. Universidad de Cantabria. Santander - España. Fraden, J. (2016). Handbook of Modern Sensors: Physics, Designs, and Applications 5th Edition. ISBN: 978-3-319-19302-1.

Manual de Construcción, Calentador de Agua Solar. Unión de Esfuerzos para el Campo, AC. (UDEC). Tequisquiapan, Qro., México.

Northrop, R. (2005). Introduction to Instrumentation and Measurements, Second Edition. ISBN: 978- 1-4200-5785-0.

Wilson J., Newnes E. (2005). Sensor Technology Handbook. ISBN: 0-7506-7729-5. 